\title{
Nanobody-Based Biologics for Modulating Purinergic Signaling in Inflammation and Immunity
}

\author{
Stephan Menzel, Nicole Schwarz, Friedrich Haag and Friedrich Koch-Nolte* \\ Institute of Immunology, University Medical Center Hamburg-Eppendorf, Hamburg, Germany
}

OPEN ACCESS

Edited by:

Kenneth A. Jacobson,

National Institutes of Health (NIH),

United States

Reviewed by:

Szilvia Benkö,

University of Debrecen, Hungary

Gregory C. Ippolito,

University of Texas at Austin,

United States

Francesco Di Virgilio,

University of Ferrara, Italy

${ }^{*}$ Correspondence:

Friedrich Koch-Nolte

nolte@uke.de

Specialty section:

This article was submitted to

Experimental Pharmacology and Drug

Discovery,

a section of the journal

Frontiers in Pharmacology

Received: 03 January 2018

Accepted: 09 March 2018

Published: 27 March 2018

Citation:

Menzel S, Schwarz N, Haag Fand

Koch-Nolte F (2018)

Nanobody-Based Biologics

for Modulating Purinergic Signaling

in Inflammation and Immunity.

Front. Pharmacol. 9:266.

doi: 10.3389/fphar.2018.00266
Adenosine triphosphate (ATP) and nicotinamide adenine dinucleotide $\left(\mathrm{NAD}^{+}\right)$are released as danger signals from cells during infection and sterile inflammation. In the extracellular compartment ATP is converted by CD39, CD73, and other ecto-enzymes into metabolites that modulate the activity of $T$ cells and macrophages. While ATP mediates pro-inflammatory signals via $\mathrm{P} 2 \mathrm{X} 7$ and other $\mathrm{P} 2$ receptors, adenosine triggers anti-inflammatory signaling via the adenosine 2a receptor (Adora2a) and other P1 receptors. The latter also plays a role in maintaining an immunosuppressive tumor microenvironment. $\mathrm{NAD}^{+}$is converted by CD38, CD203 and other ecto-enzymes to the $\mathrm{Ca}^{2+}$ mobilizing messengers cyclic ADP-ribose and ADP-ribose, and to adenosine. Recent findings on the roles of CD38, CD39, CD73, CD203, P2X7, and Adora2a in inflammation and immunity underscore the potential of these proteins as drug targets. However, available small molecule inhibitors often lack specificity and mediate unwanted off-target toxicity. Nanobodies - single domain antibodies derived from heavy chain antibodies that naturally occur in camelids - display a propensity to bind functional epitopes not accessible to conventional antibodies. Like conventional antibodies, nanobodies and nanobody-based biologics are highly specific and have well-understood, tunable in vivo pharmacodynamics with little if any toxicity. Nanobodies thus represent attractive alternatives to small molecule inhibitors for modulating purinergic signaling in inflammation and immunity. Here we review recent progress made in developing nanobodies against key targets of purinergic signaling.

Keywords: nanobody, purinergic signaling, biologics, heavy chain antibody, antibody engineering

\section{INTRODUCTION}

Purinergic signaling by extracellular ATP, $\mathrm{NAD}^{+}$and their metabolites is recognized as an important regulatory mechanism in inflammation and immunity (Junger, 2011; Eltzschig et al., 2012; Idzko et al., 2014; Burnstock, 2016). The intact nucleotides and their common metabolite adenosine play pro- and anti-inflammatory roles, respectively. The ecto-enzymes and receptors that mediate purinergic signaling, therefore, represent promising targets for immunomodulatory drugs (Burnstock, 2017). A number of small molecule inhibitors are available that antagonize

Abbreviations: ADCC, antibody-dependent cytotoxicity; ADP, adenosine diphosphate; ADPR, adenosine diphosphate ribose; AMP, adenosine monophosphate; ATP, adenosine triphosphate; $\mathrm{BBB}$, blood brain barrier; $\mathrm{CDC}$, complementdependent cytotoxicity; GPCR, G-protein coupled receptor; HLE, half-life extended; Ig, immunoglobulin, IL-, interleukin; $\mathrm{kD}$, kilodalton; $\mathrm{NAD}^{+}$, nicotinamide adenine dinucleotide; $\mathrm{NICD}, \mathrm{NAD}^{+}$induced cell death. 
ionotropic (P2X) and metabotropic (P2Y) ATP-receptors, ATPand NAD- hydrolyzing enzymes (CD38, CD39, CD73, CD203), and metabotropic (P1) adenosine receptors (Buque et al., 2016; Jacobson and Muller, 2016). Several of these show promising therapeutic benefit in animal models of inflammatory diseases and/or in animal tumor models (Buque et al., 2016; Burnstock, 2016). Recently, antibodies and nanobodies have emerged as a potent alternative class of therapeutics (Wesolowski et al., 2009; Krah et al., 2016; Steeland et al., 2016).

Nanobodies are single domain antibody fragments derived from heavy chain antibodies naturally occurring in dromedaries, llamas, and other camelids (Figure 1A) (Hamers-Casterman et al., 1993; Muyldermans, 2013). In these animals, the IgG2 and IgG3 isotypes lack light chains and the $\mathrm{CH} 1$ domain. Nanobodies correspond to the variable domain of these heavy chain antibodies (also designated VHH). VHHs carry mutations that render them highly soluble in the absence of a paired light chain. VHHs often have longer CDR3 loops than the $\mathrm{VH}$ domain of conventional antibodies. These long CDR3 loops can mediate binding to hidden epitopes on target proteins such as the catalytic cavity of an enzyme, the ligand binding site of an ion channel or of a GPCR (Lauwereys et al., 1998; De Genst et al., 2006; Wesolowski et al., 2009). Because of their small size (15 kD, $3 \mathrm{~nm}$ ), nanobodies generally show excellent tissue penetration. On the other hand, these small proteins pass the renal filtration barrier, accounting for a much shorter serum half life than that of conventional antibodies. Their robust, soluble single domain format, renders nanobodies amenable for genetic fusion to other protein domains. It is much easier to link two or more nanobodies into bi- or multivalent formats than the corresponding $\mathrm{VH}+\mathrm{VL}$ domains of conventional antibodies.
Linkage to an albumin-specific nanobody, for example, provides a simple strategy to extend the in vivo half life (Tijink et al., 2008). Nanobodies, thus represent promising tools to target the key players of purinergic signaling, in particular hidden functional epitopes of these proteins (Figure 1B).

\section{NUCLEOTIDE METABOLIZING ECTO-ENZYMES AS TARGET FOR NANOBODIES}

Adenosine triphosphate (ATP) and nicotinamide adenine dinucleotide $\left(\mathrm{NAD}^{+}\right)$are released as danger signals from cells during infection and sterile inflammation (Haag et al., 2007; Junger, 2011; Eltzschig et al., 2012; Idzko et al., 2014). In the extracellular compartment ATP is converted by CD39, CD73, CD203 and related enzymes into ADP, AMP, and adenosine, metabolites that modulate the activity of $\mathrm{T}$ cells and macrophages. $\mathrm{NAD}^{+}$is converted by CD38, CD73, and CD203 into nicotinamide and the $\mathrm{Ca}^{2+}$ mobilizing messengers cyclic ADP-ribose (cADPR) and ADP-ribose (ADPR), and finally to adenosine (Figure 1C). Extracellular $\mathrm{NAD}^{+}$is also a substrate for the CD296 family of toxin-related ecto-enzymes that modify cell surface proteins by ADP-ribosylation.

\section{Nanobodies Targeting CD296/ARTC2.2}

In response to $\mathrm{NAD}^{+}$released from cells during inflammation, the ARTC2.2 ecto-enzyme ADP-ribosylates the P2X7 ion channel (Adriouch et al., 2008; Laing et al., 2010) and other cell surface proteins. ADP-ribosylation of P2X7 at R125 covalently attaches the weak ligand ADP-ribose close to the ATP binding pocket.
A

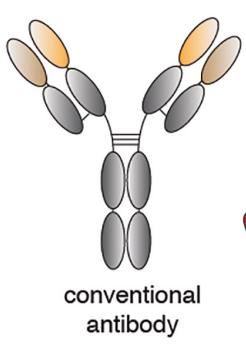

C

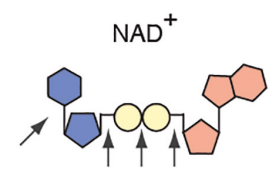

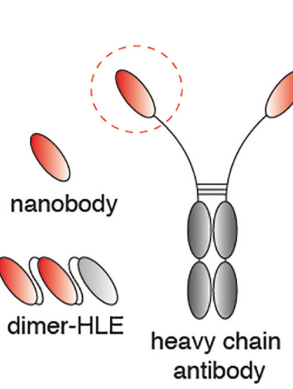

ATP

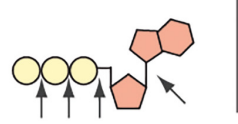

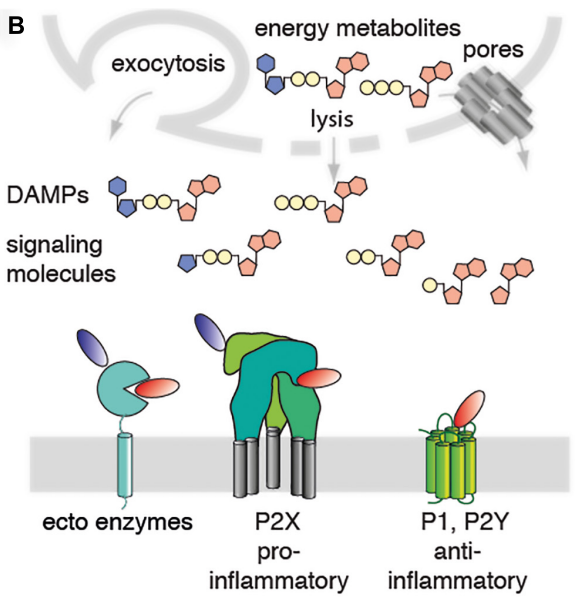

FIGURE 1 | Mediators of purinergic signaling as potential nanobody targets. (A) Nanobodies correspond to the variable domain of heavy chain antibodies. These single domain antibodies often contain a long CDR3 that can extend into crevices on proteins that are not accessible to conventional antibodies. Nanobodies are highly soluble and are thus easily linked into bivalent and/or bispecific formats. For example, fusion of a homodimeric nanobody to an albumin-specific nanobody yields a half-life extended dimer (Dimer-HLE). (B) The energy metabolites ATP and NAD ${ }^{+}$are released from cells during inflammation. In the extracellular space, these nucleotides function as danger associated molecular patterns (DAMPs) or signaling molecules. Immune cells express ecto-enzymes that convert pro-inflammatory ATP and NAD+ into anti-inflammatory adenosine. Immune cells also express ionotropic ligand-gated ion channels (P2X) and metabotropic GPCRs (P1, P2Y) that respond to ATP, NAD ${ }^{+}$and their metabolites. These ecto-enzymes and purinergic receptors represent potential targets for immunomodulatory nanobodies. Antagonist nanobodies are illustrated schematically in red, potentiating nanobodies in blue. (C) Schematic diagram of NAD ${ }^{+}$, ATP and the linkages that are targets for ecto-enzymes. 
Chronic activation of P2X7 leads to cell death. This phenomenon is designated NICD (Seman et al., 2003). Regulatory T cells, NKT cells, and tissue resident memory $\mathrm{T}$ cells (Trm) are particularly sensitive to NICD (Hubert et al., 2010; Rissiek et al., 2014; Fernandez-Ruiz et al., 2016).

ARTC2 ecto-enzymes are not expressed by human cells because the orthologous gene in humans and other primates is inactivated by premature stop codons (Haag et al., 1994). Notwithstanding, ARTC2.2 is the first immune cell ecto-enzyme for which potent, highly specific, antagonistic nanobodies have been developed (Koch-Nolte et al., 2007). Systemic application of ARTC2.2 -blocking nanobodies in mouse models have yielded important insights that may be relevant also to nanobodies targeting other mediators of purinergic signaling. Nanobodies reach and opsonize ARTC2.2 on the surface of immune cells much faster than conventional antibodies: After intravenous or intraperitoneal injection at moderate dosage $(2 \mathrm{mg} / \mathrm{kg})$, the nanobodies completely opsonized ARTC2.2 within 10 min after injection, whereas conventional antibodies achieved this only at $2 \mathrm{~h}$ after injection (Koch-Nolte et al., 2007; Hubert et al., 2010; Scheuplein et al., 2010). Conversely, unbound nanobodies were rapidly eliminated via renal infiltration while conventional antibodies and nanobody-based heavy chain antibodies were shown to have a much longer half life (Bannas et al., 2014, 2015). The effective functional blockade of ARTC2.2 enzyme activity by the injected nanobodies and nanobody-based heavy chain antibodies could readily be monitored on $\mathrm{T}$ cells recovered from lymphatic tissues of nanobody-injected mice: both formats effectively blocked ARTC2.2-catalyzed ADP-ribosylation of cell surface proteins and ARTC2.2-mediated NICD (Koch-Nolte et al., 2007; Hubert et al., 2010; Scheuplein et al., 2010). By introducing three amino acid substitutions into the Fc domain [LSF previously shown to mediate enhanced binding of IgG to the neonatal Fc-receptor (Ghetie et al., 1997)] blockade of ARTC2.2 in vivo could be extended to $>1$ week, even after injection of only a very low dose $(0.2 \mathrm{mg} / \mathrm{kg})$ of the nanobody-based heavy chain antibody LSF mutant (Scheuplein et al., 2010). In the NOD mouse model of genetically determined type 1 diabetes, weekly injections of this ARTC2.2-blocking heavy chain antibody resulted in a significant elevation of iNKT cell numbers in lymph nodes and spleen for at least 4 weeks after injection.

During the preparation of primary lymphocytes from spleen, lymph node, blood or liver, sufficient quantities of $\mathrm{NAD}^{+}$are released from the mechanically stressed cells to permit ARTC2.2catalyzed ADP-ribosylation of P2X7 and induction of NICD of Tregs, NKT, and Trm cells (Hubert et al., 2010; Rissiek et al., 2014; Fernandez-Ruiz et al., 2016). A single systemic injection of ARTC2.2-blocking nanobodies or nanobody-based heavy chain antibodies $30 \mathrm{~min}$ before sacrifice effectively prevents NICD of NKT cells and Tregs during cell preparation. Without such treatment ARTC2.2-expressing regulatory immune cells show poor survival during in vitro re-stimulation as well in adoptive transfer studies.

\section{Nanobodies Targeting CD38}

$\mathrm{CD} 38$, the major $\mathrm{NAD}^{+}$-hydrolyzing ecto-enzyme, plays a decisive role in controlling the local levels of extracellular
$\mathrm{NAD}^{+}$. CD38 is expressed by various hematopoietic cells, in particular by $\mathrm{B}$ cells and NK cells. CD38 hydrolyzes $\mathrm{NAD}^{+}$to ADPR and CADPR, releasing nicotinamide. ADPribose is further hydrolyzed to phosphoriboside and AMP by members of the CD203 family of phosphodiesterases and eventually to adenosine by CD73. Two CD38-specific antibodies (daratumumab, isatuximab) have recently been licensed for the treatment of multiple myeloma, since these tumor cells often upregulate cell surface expression of CD38 to very high levels (van de Donk et al., 2017). The therapeutic benefit of these antibodies is thought to be mediated by their cytotoxic effects on tumor cells rather than by antagonizing the enzymatic activity of CD38. Recent evidence indicates that CD38 is also strongly upregulated by many solid tumors. It has been proposed that CD38 contributes to the immunosuppressive tumor microenvironment by hydrolyzing $\mathrm{NAD}^{+}$(Horenstein et al., 2015). In line with this hypothesis, blocking CD38 enzyme activity in the tumor microenvironment may be a therapeutic strategy. Isatuximab was shown to inhibit the enzymatic activity of CD38, even though its binding site is far away from the $\mathrm{NAD}^{+}$binding crevice, implying that this antibody acts by an allosteric mechanism (Deckert et al., 2014). Recently, 22 CD38specific nanobodies were reported, two of which inhibited and two of which enhanced CD38 enzymatic activity (Li et al., 2016; Fumey et al., 2017). The results of epitope mapping and cocrystallization analyses indicate that these functional nanobodies, like isatuximab, act in an allosteric fashion rather than by direct binding to the active site. It is conceivable that the active site cleft of CD38 faces the plasma membrane and therefore is not easily accessible to antibodies or nanobodies. Some of the nanobodies have been shown to effectively target CD38 on human tumor cells in a mouse Xenograft model (Fumey et al., 2017) and some of these nanobodies bind independently of daratumumab and isatuximab and may, therefore, be useful for detecting cell surface CD38 in daratumumab- and isatuximabtreated patients (Oberle et al., 2017).

\section{Other Ecto-Enzymes as Potential Targets for Inhibitory Nanobodies}

Similar to ARTC2.2 and CD38, other nucleotide metabolizing ecto-enzymes including CD39, CD73, CD203, and alkaline phosphatase represent promising targets for nanobody-based antagonists and/or agonists (Yegutkin, 2008; Deaglio and Robson, 2011; Horenstein et al., 2013; al-Rashida and Iqbal, 2014) Blocking the hydrolysis of nucleotides to adenosine is predicted to promote inflammatory responses and thus may represent viable therapeutic strategies in chronic infection and oncology. Conversely, promoting the conversion of extracellular nucleotides to adenosine may be beneficial in chronic inflammation. Many of these enzymes display deep active site crevices that are difficult to block with conventional antibodies but may be accessible for nanobodies. CD73 may present an exception from this theme. The crystal structures of CD73 in closed and open conformation revealed that this enzyme undergoes a dramatic conformational rearrangement upon AMP-binding (Knapp et al., 2012). This dramatic 
conformational shift may facilitate antibody mediated inhibition of CD73 by non-competitive mechanisms, e.g., by sterically blocking the shift in conformation, rather than occluding the active site (Geoghegan et al., 2016).

\section{PURINERGIC P1 AND P2 RECEPTORS AS TARGETS FOR NANOBODIES}

ATP, $\mathrm{NAD}^{+}$and their metabolites act as ligands for ionotropic (P2X) and metabotropic (P1, P2Y) receptors, many of which are expressed by immune cells (Burnstock and Boeynaems, 2014; Di Virgilio and Vuerich, 2015). Targeting these cell surface receptors with antagonistic and/or agonistic nanobodies thus might also provide novel therapeutic approaches in inflammation and immunity.

\section{Nanobodies Targeting P2X7}

The P2X7 ion channel is expressed by monocytes and T cells and is gated upon binding of extracellular ATP, permitting influx of calcium $\left(\mathrm{Ca}^{2+}\right)$ and sodium $\left(\mathrm{Na}^{+}\right)$and efflux of potassium $\left(\mathrm{K}^{+}\right)$(Bartlett et al., 2014; Di Virgilio et al., 2017). This triggers assembly of the inflammasome, release of the pro-inflammatory cytokine IL-1 $\beta$ (IL-1 $\beta$ ), ectodomain shedding of membrane proteins and externalization of phosphatidylserine (Ferrari et al., 2006; Giuliani et al., 2017). P2X7 is a potential therapeutic target in inflammatory diseases, such as glomerulonephritis, multiple sclerosis, and chronic pain (Bartlett et al., 2014; McInnes et al., 2014).

A set of 21 nanobodies was selected by phage display from llamas immunized with cDNA expression vectors encoding mouse and human P2X7 (Danquah et al., 2016). Six of eighteen nanobody families either blocked or enhanced activation of mouse P2X7 by both ATP and $\mathrm{NAD}^{+}$-mediated pathways and two of three nanobody families effectively blocked ATPmediated gating of human P2X7. Dimerization via a flexible G4S linker enhanced the potencies of both, the antagonistic 13A7 nanobody and the potentiating 14D5 nanobody to low nanomolar/high picomolar $\mathrm{IC}_{50}$ values. Both nanobodies modulated P2X7 function when added either before or after the ligand ATP. Intriguingly, addition of the $13 \mathrm{~A} 7$ blocked binding of the $14 \mathrm{D} 5$ and vice versa, indicating that these nanobodies either bind to overlapping epitopes or to alternative conformational states of P2X7. Genetic fusion of homodimeric nanobodies to the albumin-binding nanobody Alb8 markedly increased the in vivo half-life from hours to days. This trimeric nanobody-format was designated HLE-dimer (half-life extended $\alpha \mathrm{P} 2 \mathrm{X} 7$ dimer). A similar extension of the in vivo half life was achieved by fusing the nanobody monomers to the hinge and Fc domains of mouse or human IgG, thereby reconstituting a bivalent heavy chain antibody format. In two different mouse models systemic administration of the P2X7-antagonistic 13A7 HLE-dimer at a moderate dosage (1 $\mathrm{mg} / \mathrm{kg}$ every 3 days) significantly ameliorated inflammation scores. In the model of DNTB-induced allergic contact dermatitis, 13A7 HLE-dimer reduced local inflammation as measured by ear weight and levels of the inflammatory cytokines IL-1ß and IL-6 to a similar extent as the corticosteroid Dexamethasone. Similarly, in the model of antibody-induced glomerulonephritis, the P2X7antagonistic 13A7 HLE-dimer significantly reduced leukocyte infiltration of glomeruli and proteinuria. In a surrogate human inflammation model with endotoxin-treated blood samples, the human P2X7 antagonistic Dano1 nanobody effectively blocked ATP-induced release of IL- $1 \beta$ with subnanomolar IC $_{50}$ values, i.e., at $>1.000$ fold higher potency than benchmark small molecule inhibitors of P2X7. Nanobody Dano1 thus is an excellent clinical candidate.

\section{Other Purinergic Receptors as Potential Targets for Inhibitory Nanobodies}

Analogous to P2X7, P2X4, and P2X1 are expressed by immune cells and could be targets for nanobody-based antagonists and/or agonists (Burnstock, 2016). The P1 and P2Y receptors are GPCRs that respond to adenosine and purine nucleotides, respectively (Jacobson and Muller, 2016) and Adora2a and P2Y11 receptors are most relevant for inflammation and immunity (Sitkovsky et al., 2014; Young et al., 2016; Kennedy, 2017). Since these GPCRs have much smaller extracellular domains than the P2X receptors, inducing specific antibodies against GPCRs is much more challenging than in case of P2XRs. Notwithstanding, potent antagonistic nanobodies have been developed from llamas immunized with cDNA expression vectors encoding two nonpurinergic lymphocyte GPCRs: CXCR4 and ChemR23, receptors for chemotactic proteins (Jahnichen et al., 2010; Claes et al., 2016; Peyrassol et al., 2016). It is thus not farfetched to propose that this strategy may also yield nanobodies targeting P1 and P2Y receptors.

\section{ADVANTAGES AND LIMITATIONS OF NANOBODY-BASED BIOLOGICS IN PURINERGIC PHARMACOLOGY}

Nanobodies directed against key players of purinergic signaling are valuable tools for research and diagnosis. Fluorochromeconjugated nanobodies, for example, are well suited for flow cytometry, microscopy, and molecular imaging applications (Fumey et al., 2017). For example, nanobody JK36 recognizes a non-overlapping epitope on CD38 and can therefore detect cell surface CD38 by flow cytometry even in patients under treatment with daratumumab - where occupancy of CD38 by daratumumab prevents binding of most commercial diagnostic mAbs (Oberle et al., 2017). Owing to their small size, fluorochrome-conjugated nanobodies often provide much higher resolution images in fluorescence microscopy than conventional antibodies (Pleiner et al., 2015). Nanobodies have also proven useful as crystallization chaperones for 3D-structure analyses of GPCRs (Steyaert and Kobilka, 2011). On the other hand, native membrane protein-specific nanobodies usually do not work well in Western-Blot analyses, since their target epitopes are often destroyed by denaturing gel electrophoresis.

Table 1 summarizes advantages and limitations of nanobodies vs. antibodies and small molecule drugs. In contrast to most small molecule drugs, antibodies and nanobodies generally are 
TABLE 1 | Advantages and limitations of nanobodies and antibodies vs. small molecule drugs in purinergic pharmacology.

\begin{tabular}{|c|c|c|c|}
\hline & Antibodies & Nanobodies & Small molecules \\
\hline Size & 150 kD & $15 \mathrm{kD}$ & $\sim 1 \mathrm{kD}$ \\
\hline Development costs & High & Moderate & Usually low \\
\hline Administration & i.v., s.c. & i.v., s.c., aerosol, topical & oral, i.v. \\
\hline Specificity & High & High & Variable \\
\hline Off target adverse effects & None & None & P1, P2, kinases, ATPases, dehydrogenases \\
\hline On target adverse effects & Depends on target & Depends on target & Depends on target \\
\hline In Vivo half-life & Can be adjusted by Fc-engineering & $\begin{array}{l}\text { Can be adjusted by PEGylation or fusion to } \\
\text { albumin-specific } \mathrm{Nb}\end{array}$ & Variable (usually short) \\
\hline Metabolites & Non-toxic, biodegradable & Non-toxic, biodegradable & Potentially toxic \\
\hline Tissue penetration & Slow & Excellent in periphery & Variable \\
\hline Tissue specificity & Targetable (bi-specific Abs) & Targetable (bi- specific Nbs) & Variable \\
\hline Albumin binding & Usually not & $\begin{array}{l}\text { Via albumin-specific Nb to extend half life, } \\
\text { usually no effect on potency }\end{array}$ & May reduce potency \\
\hline
\end{tabular}

not orally available, i.e., they need to be administered by infusion or subcutaneous injection. High costs of clinical development and production still are handicaps of antibodies and nanobodies (Brekke and Sandlie, 2003). On the other hand, antibodies and nanobodies show exquisite specificity to the target molecule and in contrast to small molecules - show little if any off-target side effects (Steeland et al., 2016). Nanobodies and antibodies are biodegradable and - in contrast to small molecules - do not suffer from conversion to toxic metabolites. While antibodies display uniform pharmacodynamics and a longer in vivo or serum half life than small molecules and nanobodies, their large size $(150 \mathrm{kD})$ limits their penetration into solid tumors and other tissues. With a small size $(15 \mathrm{kD})$, excellent tissue penetration and rapid renal filtration of unbound molecules, nanobodies, however, are exceptionally well suited for in vivo molecular imaging applications (Chakravarty et al., 2014; Bannas et al., 2015; Rashidian et al., 2015; Beghein and Gettemans, 2017).

Antibodies and nanobodies both show little if any capacity to cross the $\mathrm{BBB}$. This can be an advantage for the therapeutic targeting of purinergic enzymes and receptors in inflammation and oncology outside the nervous system, because this limits on-target side effects in the central nervous system. The $\mathrm{BBB}$, however, presents a limitation for therapeutic targeting inflammation and oncology in the CNS. If desired, passage of antibodies through the $\mathrm{BBB}$ can be enhanced by genetic fusion or conjugation to binding modules for the transferrin receptor and other transcytosis receptors (Farrington et al., 2014). Because of their small size and modular nature, nanobodies may be suited better for this strategy than conventional antibodies. The Fc-domain of conventional antibodies can mediate unwanted complement dependent and/or cell-mediated cytotoxicity (CDC, ADCC). Here, lack of an Fc domain can be an advantage for nanobodies. If the deletion of tumor cells or certain immune cell subsets is desired for therapeutic purposes, nanobodies can readily be fused to the Fc domain of conventional antibodies to generate a nanobodybased heavy chain antibody $(75 \mathrm{kD})$. Such heavy chain antibodies are amenable for the full power of Fc-engineering technologies, i.e., substitution of amino acid residues that enhance/reduce in vivo half life and/or cytotoxicity (Liu et al., 2017).

Most purine metabolizing ecto-enzymes and purinergic receptors are not restricted to immune cells. Many of these enzymes and receptors are also expressed by cells of the cardiovascular system and the CNS. It will thus be important to monitor potential on-target side effects of the nanobodies that modulate their target on cells of other tissues. Owing to their high solubility and modular format, nanobodies can easily be linked to other nanobodies (Els Conrath et al., 2001; Bannas et al., 2017). This opens the opportunity to generate bi-specific biologics with improved cellular specificity, e.g., by linking a purinergic receptor-specific nanobody to a nanobody directed against a tissue differentiation antigen.

\section{CONCLUSION AND OUTLOOK}

Nanobodies display a propensity to bind functional epitopes not accessible to conventional antibodies. Nanobodies that antagonize the purine-metabolizing ecto-enzymes ARTC2.2 and CD38 or the ATP-gated ion channel P2X7 have provided proof of concept for the notion that these small biologics represent attractive alternatives to small molecule inhibitors for modulating purinergic signaling in inflammation and immunity. It is thus not unlikely that caplacizumab, the first nanobody that is bound to reach the clinic next year, will pave the way for nanobody-based therapeutics also in the field of purinergic pharmacology.

\section{AUTHOR CONTRIBUTIONS}

FH and FK-N conceived the project. SM, NS, and FK-N wrote the manuscript. All authors reviewed and approved the manuscript.

\section{FUNDING}

This work was supported by Grant Nos. 310/11-1, SFB1192 B5, and Ha2369/5-1 to FK-N and FH. 


\section{REFERENCES}

Adriouch, S., Bannas, P., Schwarz, N., Fliegert, R., Guse, A. H., Seman, M., et al. (2008). ADP-ribosylation at R125 gates the P2X7 ion channel by presenting a covalent ligand to its nucleotide binding site. FASEB J. 22, 861-869. doi: 10.1096/fj.07-9294com

al-Rashida, M., and Iqbal, J. (2014). Therapeutic potentials of ectonucleoside triphosphate diphosphohydrolase, ecto-nucleotide pyrophosphatase/phosphodiesterase, ecto-5'-nucleotidase, and alkaline phosphatase inhibitors. Med. Res. Rev. 34, 703-743. doi: 10.1002/med.21302

Bannas, P., Hambach, J., and Koch-Nolte, F. (2017). Nanobodies and nanobodybased human heavy chain antibodies as antitumor therapeutics. Front. Immunol. 8:1603. doi: 10.3389/fimmu.2017.01603

Bannas, P., Lenz, A., Kunick, V., Well, L., Fumey, W., Rissiek, B., et al. (2015). Molecular imaging of tumors with nanobodies and antibodies: timing and dosage are crucial factors for improved in vivo detection. Contrast Media Mol. Imaging 10, 367-378. doi: 10.1002/cmmi.1637

Bannas, P., Well, L., Lenz, A., Rissiek, B., Haag, F., Schmid, J., et al. (2014). In vivo near-infrared fluorescence targeting of T cells: comparison of nanobodies and conventional monoclonal antibodies. Contrast Media Mol. Imaging 9, 135-142. doi: $10.1002 / \mathrm{cmmi} .1548$

Bartlett, R., Stokes, L., and Sluyter, R. (2014). The P2X7 receptor channel: recent developments and the use of P2X7 antagonists in models of disease. Pharmacol. Rev. 66, 638-675. doi: 10.1124/pr.113.008003

Beghein, E., and Gettemans, J. (2017). Nanobody technology: a versatile toolkit for microscopic imaging, protein-protein interaction analysis, and protein function exploration. Front. Immunol. 8:771. doi: 10.3389/fimmu.2017.0 0771

Brekke, O. H., and Sandlie, I. (2003). Therapeutic antibodies for human diseases at the dawn of the twenty-first century. Nat. Rev. Drug Discov. 2, 52-62. doi: $10.1038 /$ nrd984

Buque, A., Bloy, N., Aranda, F., Cremer, I., Eggermont, A., Fridman, W. H., et al. (2016). Trial watch-small molecules targeting the immunological tumor microenvironment for cancer therapy. Oncoimmunology 5:e1149674. doi: 10. 1080/2162402X.2016.1149674

Burnstock, G. (2016). P2X ion channel receptors and inflammation. Purinergic Signal. 12, 59-67. doi: 10.1007/s11302-015-9493-0

Burnstock, G. (2017). The therapeutic potential of purinergic signalling. Biochem. Pharmacol. doi: 10.1016/j.bcp.2017.07.016 [Epub ahead of print].

Burnstock, G., and Boeynaems, J. M. (2014). Purinergic signalling and immune cells. Purinergic Signal. 10, 529-564. doi: 10.1007/s11302-014-9427-2

Chakravarty, R., Goel, S., and Cai, W. (2014). Nanobody: the "magic bullet" for molecular imaging? Theranostics 4, 386-398. doi: 10.7150/thno.8006

Claes, K., Vandewalle, K., Laukens, B., Laeremans, T., Vosters, O., Langer, I., et al. (2016). Modular integrated secretory system engineering in pichia pastoris to enhance G-protein coupled receptor expression. ACS Synth. Biol. 5, 1070-1075. doi: 10.1021/acssynbio.6b00032

Danquah, W., Meyer-Schwesinger, C., Rissiek, B., Pinto, C., Serracant-Prat, A., Amadi, M., et al. (2016). Nanobodies that block gating of the P2X7 ion channel ameliorate inflammation. Sci. Transl. Med. 8:366ra162. doi: 10.1126/ scitranslmed.aaf8463

De Genst, E., Silence, K., Decanniere, K., Conrath, K., Loris, R., Kinne, J., et al. (2006). Molecular basis for the preferential cleft recognition by dromedary heavy-chain antibodies. Proc. Natl. Acad. Sci. U.S.A. 103, 4586-4591. doi: 10.1073/pnas.0505379103

Deaglio, S., and Robson, S. C. (2011). Ectonucleotidases as regulators of purinergic signaling in thrombosis, inflammation, and immunity. Adv. Pharmacol. 61, 301-332. doi: 10.1016/B978-0-12-385526-8.00010-2

Deckert, J., Wetzel, M. C., Bartle, L. M., Skaletskaya, A., Goldmacher, V. S., Vallee, F., et al. (2014). SAR650984, a novel humanized CD38-targeting antibody, demonstrates potent antitumor activity in models of multiple myeloma and other CD38+ hematologic malignancies. Clin. Cancer Res. 20, 4574-4583. doi: 10.1158/1078-0432.CCR-14-0695

Di Virgilio, F., Dal Ben, D., Sarti, A. C., Giuliani, A. L., and Falzoni, S. (2017). The P2X7 receptor in infection and inflammation. Immunity 47, 15-31. doi: 10.1016/j.immuni.2017.06.020

Di Virgilio, F., and Vuerich, M. (2015). Purinergic signaling in the immune system. Auton. Neurosci. 191, 117-123. doi: 10.1016/j.autneu.2015.04.011
Els Conrath, K., Lauwereys, M., Wyns, L., and Muyldermans, S. (2001). Camel single-domain antibodies as modular building units in bispecific and bivalent antibody constructs. J. Biol. Chem. 276, 7346-7350. doi: 10.1074/jbc. M007734200

Eltzschig, H. K., Sitkovsky, M. V., and Robson, S. C. (2012). Purinergic signaling during inflammation. N. Engl. J. Med. 367, 2322-2333. doi: 10.1056/ NEJMra1205750

Farrington, G. K., Caram-Salas, N., Haqqani, A. S., Brunette, E., Eldredge, J., Pepinsky, B., et al. (2014). A novel platform for engineering blood-brain barriercrossing bispecific biologics. FASEB J. 28, 4764-4778. doi: 10.1096/fj.14-253369

Fernandez-Ruiz, D., Ng, W. Y., Holz, L. E., Ma, J. Z., Zaid, A., Wong, Y. C., et al. (2016). Liver-resident memory CD8(+) T cells form a front-line defense against malaria liver-stage infection. Immunity 45, 889-902. doi: 10.1016/j.immuni. 2016.08.011

Ferrari, D., Pizzirani, C., Adinolfi, E., Lemoli, R. M., Curti, A., Idzko, M., et al. (2006). The P2X7 receptor: a key player in IL-1 processing and release. J. Immunol. 176, 3877-3883. doi: 10.4049/jimmunol.176.7.3877

Fumey, W., Koenigsdorf, J., Kunick, V., Menzel, S., Schutze, K., Unger, M., et al. (2017). Nanobodies effectively modulate the enzymatic activity of CD38 and allow specific imaging of CD38(+) tumors in mouse models in vivo. Sci. Rep. 7:14289. doi: 10.1038/s41598-017-14112-6

Geoghegan, J. C., Diedrich, G., Lu, X., Rosenthal, K., Sachsenmeier, K. F., $\mathrm{Wu}, \mathrm{H}$., et al. (2016). Inhibition of CD73 AMP hydrolysis by a therapeutic antibody with a dual, non-competitive mechanism of action. mAbs 8, 454-467. doi: 10.1080/19420862.2016.1143182

Ghetie, V., Popov, S., Borvak, J., Radu, C., Matesoi, D., Medesan, C., et al. (1997). Increasing the serum persistence of an $\mathrm{IgG}$ fragment by random mutagenesis. Nat. Biotechnol. 15, 637-640. doi: 10.1038/nbt0797-637

Giuliani, A. L., Sarti, A. C., Falzoni, S., and Di Virgilio, F. (2017). The P2X7 Receptor-Interleukin-1 Liaison. Front. Pharmacol. 8:123. doi: 10.3389/fphar. 2017.00123

Haag, F., Adriouch, S., Brass, A., Jung, C., Moller, S., Scheuplein, F., et al. (2007). Extracellular NAD and ATP: partners in immune cell modulation. Purinergic Signal. 3, 71-81. doi: 10.1007/s11302-006-9038-7

Haag, F., Koch-Nolte, F., Kuhl, M., Lorenzen, S., and Thiele, H. G. (1994). Premature stop codons inactivate the RT6 genes of the human and chimpanzee species. J. Mol. Biol. 243, 537-546. doi: 10.1006/jmbi.1994.1680

Hamers-Casterman, C., Atarhouch, T., Muyldermans, S., Robinson, G., Hamers, C., Songa, E. B., et al. (1993). Naturally occurring antibodies devoid of light chains. Nature 363, 446-448. doi: 10.1038/363446a0

Horenstein, A. L., Chillemi, A., Quarona, V., Zito, A., Roato, I., Morandi, F., et al. (2015). $\mathrm{NAD}(+)$-metabolizing ectoenzymes in remodeling tumor-host interactions: the human myeloma model. Cells 4, 520-537. doi: 10.3390/ cells 4030520

Horenstein, A. L., Chillemi, A., Zaccarello, G., Bruzzone, S., Quarona, V., Zito, A., et al. (2013). A CD38/CD203a/CD73 ectoenzymatic pathway independent of CD39 drives a novel adenosinergic loop in human $\mathrm{T}$ lymphocytes. Oncoimmunology 2:e26246. doi: 10.4161/onci.26246

Hubert, S., Rissiek, B., Klages, K., Huehn, J., Sparwasser, T., Haag, F., et al. (2010). Extracellular NAD+ shapes the Foxp3+ regulatory T cell compartment through the ART2-P2X7 pathway. J. Exp. Med. 207, 2561-2568. doi: 10.1084/ jem.20091154

Idzko, M., Ferrari, D., and Eltzschig, H. K. (2014). Nucleotide signalling during inflammation. Nature 509, 310-317. doi: 10.1038/nature13085

Jacobson, K. A., and Muller, C. E. (2016). Medicinal chemistry of adenosine, P2Y and P2X receptors. Neuropharmacology 104, 31-49. doi: 10.1016/j.neuropharm. 2015.12.001

Jahnichen, S., Blanchetot, C., Maussang, D., Gonzalez-Pajuelo, M., Chow, K. Y., Bosch, L., et al. (2010). CXCR4 nanobodies (VHH-based single variable domains) potently inhibit chemotaxis and HIV-1 replication and mobilize stem cells. Proc. Natl. Acad. Sci. U.S.A. 107, 20565-20570. doi: 10.1073/pnas. 1012865107

Junger, W. G. (2011). Immune cell regulation by autocrine purinergic signalling. Nat. Rev. Immunol. 11, 201-212. doi: 10.1038/nri2938

Kennedy, C. (2017). P2Y11 receptors: properties. distribution and functions. Adv. Exp. Med. Biol. 1051, 107-122. doi: 10.1007/5584_2017_89

Knapp, K., Zebisch, M., Pippel, J., El-Tayeb, A., Muller, C. E., and Strater, N. (2012). Crystal structure of the human ecto-5'-nucleotidase (CD73): insights into the 
regulation of purinergic signaling. Structure 20, 2161-2173. doi: 10.1016/j.str. 2012.10.001

Koch-Nolte, F., Reyelt, J., Schossow, B., Schwarz, N., Scheuplein, F., Rothenburg, S., et al. (2007). Single domain antibodies from llama effectively and specifically block $\mathrm{T}$ cell ecto-ADP-ribosyltransferase ART2.2 in vivo. FASEB J. 21, 3490-3498. doi: 10.1096/fj.07-8661com

Krah, S., Schroter, C., Zielonka, S., Empting, M., Valldorf, B., and Kolmar, H. (2016). Single-domain antibodies for biomedical applications. Immunopharmacol. Immunotoxicol. 38, 21-28. doi: 10.3109/08923973.2015. 1102934

Laing, S., Unger, M., Koch-Nolte, F., and Haag, F. (2010). ADP-ribosylation of arginine. Amino Acids 41, 257-269. doi: 10.1007/s00726-010-0676-2

Lauwereys, M., Arbabi Ghahroudi, M., Desmyter, A., Kinne, J., Holzer, W., De Genst, E., et al. (1998). Potent enzyme inhibitors derived from dromedary heavy-chain antibodies. EMBO J. 17, 3512-3520. doi: 10.1093/emboj/17.13. 3512

Li, T., Qi, S., Unger, M., Hou, Y. N., Deng, Q. W., Liu, J., et al. (2016). Immunotargeting the multifunctional CD38 using nanobody. Sci. Rep. 6:27055. doi: $10.1038 /$ srep 27055

Liu, H., Saxena, A., Sidhu, S. S., and Wu, D. (2017). Fc engineering for developing therapeutic bispecific antibodies and novel scaffolds. Front. Immunol. 8:38. doi: 10.3389/fimmu.2017.00038

McInnes, I. B., Cruwys, S., Bowers, K., and Braddock, M. (2014). Targeting the $\mathrm{P} 2 \mathrm{X} 7$ receptor in rheumatoid arthritis: biological rationale for P2X7 antagonism. Clin. Exp. Rheumatol. 32, 878-882.

Muyldermans, S. (2013). Nanobodies: natural single-domain antibodies. Annu. Rev. Biochem. 82, 775-797. doi: 10.1146/annurev-biochem-063011-092449

Oberle, A., Brandt, A., Alawi, M., Langebrake, C., Janjetovic, S., Wolschke, C., et al. (2017). Long-term CD38 saturation by daratumumab interferes with diagnostic myeloma cell detection. Haematologica 102, e368-e370. doi: 10.3324/haematol. 2017.169235

Peyrassol, X., Laeremans, T., Gouwy, M., Lahura, V., Debulpaep, M., Van Damme, J., et al. (2016). Development by genetic immunization of monovalent antibodies (nanobodies) behaving as antagonists of the human ChemR23 receptor. J. Immunol. 196, 2893-2901. doi: 10.4049/jimmunol.1500888

Pleiner, T., Bates, M., Trakhanov, S., Lee, C. T., Schliep, J. E., Chug, H., et al. (2015). Nanobodies: site-specific labeling for super-resolution imaging, rapid epitope-mapping and native protein complex isolation. eLife 4:e11349. doi: 10.7554/eLife.11349

Rashidian, M., Keliher, E. J., Bilate, A. M., Duarte, J. N., Wojtkiewicz, G. R., Jacobsen, J. T., et al. (2015). Noninvasive imaging of immune responses. Proc. Natl. Acad. Sci. U.S.A. 112, 6146-6151. doi: 10.1073/pnas.1502609112

Rissiek, B., Danquah, W., Haag, F., and Koch-Nolte, F. (2014). Technical advance: a new cell preparation strategy that greatly improves the yield of vital and functional Tregs and NKT cells. J. Leukoc. Biol. 95, 543-549. doi: 10.1189/jlb. 0713407

Scheuplein, F., Rissiek, B., Driver, J. P., Chen, Y. G., Koch-Nolte, F., and Serreze, D. V. (2010). A recombinant heavy chain antibody approach blocks ART2 mediated deletion of an iNKT cell population that upon activation inhibits autoimmune diabetes. J. Autoimmun. 34, 145-154. doi: 10.1016/j.jaut.2009.0 8.012
Seman, M., Adriouch, S., Scheuplein, F., Krebs, C., Freese, D., Glowacki, G., et al. (2003). NAD-induced T cell death: ADP-ribosylation of cell surface proteins by ART2 activates the cytolytic P2X7 purinoceptor. Immunity 19, 571-582. doi: 10.1016/S1074-7613(03)00266-8

Sitkovsky, M. V., Hatfield, S., Abbott, R., Belikoff, B., Lukashev, D., and Ohta, A. (2014). Hostile, hypoxia-A2-adenosinergic tumor biology as the next barrier to overcome for tumor immunologists. Cancer Immunol. Res. 2, 598-605. doi: 10.1158/2326-6066.CIR-14-0075

Steeland, S., Vandenbroucke, R. E., and Libert, C. (2016). Nanobodies as therapeutics: big opportunities for small antibodies. Drug Discov. Today 21, 1076-1113. doi: 10.1016/j.drudis.2016.04.003

Steyaert, J., and Kobilka, B. K. (2011). Nanobody stabilization of G proteincoupled receptor conformational states. Curr. Opin. Struct. Biol. 21, 567-572. doi: 10.1016/j.sbi.2011.06.011

Tijink, B. M., Laeremans, T., Budde, M., Stigter-van Walsum, M., Dreier, T., de Haard, H. J., et al. (2008). Improved tumor targeting of anti-epidermal growth factor receptor nanobodies through albumin binding: taking advantage of modular Nanobody technology. Mol. Cancer Ther. 7, 2288-2297. doi: 10. 1158/1535-7163.MCT-07-2384

van de Donk, N., Richardson, P. G., and Malavasi, F. (2017). CD38 antibodies in multiple myeloma: back to the future. Blood 131, 13-29. doi: 10.1182/blood2017-06-740944

Wesolowski, J., Alzogaray, V., Reyelt, J., Unger, M., Juarez, K., Urrutia, M., et al. (2009). Single domain antibodies: promising experimental and therapeutic tools in infection and immunity. Med. Microbiol. Immunol. 198, 157-174. doi: 10.1007/s00430-009-0116-7

Yegutkin, G. G. (2008). Nucleotide- and nucleoside-converting ectoenzymes: important modulators of purinergic signalling cascade. Biochim. Biophys. Acta 1783, 673-694. doi: 10.1016/j.bbamcr.2008.0 1.024

Young, A., Ngiow, S. F., Barkauskas, D. S., Sult, E., Hay, C., Blake, S. J., et al. (2016). Co-inhibition of CD73 and A2AR adenosine signaling improves antitumor immune responses. Cancer Cell 30, 391-403. doi: 10.1016/j.ccell.2016.0 6.025

Conflict of Interest Statement: FH and FK-N receive a share of antibody sales via MediGate $\mathrm{GmbH}$, a wholly owned subsidiary of the University Medical Center Hamburg-Eppendorf. SM and FK-N are co-inventors on patent applications on nanobody transgenic mice and/or CD38- or P2X7-specific nanobodies.

The other author declares that the research was conducted in the absence of any commercial or financial relationships that could be construed as a potential conflict of interest.

Copyright (C) 2018 Menzel, Schwarz, Haag and Koch-Nolte. This is an open-access article distributed under the terms of the Creative Commons Attribution License (CC BY). The use, distribution or reproduction in other forums is permitted, provided the original author(s) and the copyright owner are credited and that the original publication in this journal is cited, in accordance with accepted academic practice. No use, distribution or reproduction is permitted which does not comply with these terms. 